\title{
Qualitative behavior of a host-pathogen model
}

\author{
Qamar Din*, Abdul Qadeer Khan and Muhammad Naeem Qureshi
}

"Correspondence: qamar.sms@gmail.com

Department of Mathematics, University of Azad Jammu and Kashmir, Muzaffarabad, Pakistan

\begin{abstract}
In this paper, we study the qualitative behavior of a discrete-time host-pathogen model for spread of an infectious disease with permanent immunity. The time-step is equal to the duration of the infectious phase. Moreover, the local asymptotic stability, the global behavior of unique positive equilibrium point, and the rate of convergence of positive solutions is discussed. Some numerical examples are given to verify our theoretical results.
\end{abstract}

MSC: 39A10; 40A05

Keywords: difference equations; local stability; global character

\section{Introduction}

It is a well-known fact that in the population growth, the disease is an important agent controlling the population dynamics. Many experiments show that parasites can reasonably reduce the host population and even take the host population to complete annihilation. This natural phenomenon is successfully modeled by many simple SI type host-parasite models. The most interesting properties of such models are their ability of generating host annihilation dynamics with the ideal parametric values and initial conditions. This is possible, because such models naturally contain the proportion transmission term, which is often referred to as ratio-dependent functional response in the case of predator-prey models. In the $S I$ model, the population is subdivided into two classes, susceptibles $S$ and infectives $I$. The notation $S I$ means that there is a transfer from the susceptible to infective class, susceptibles become infective and do not recover from the infection. Thus, the transfer continues until all individuals become infected. This type of model is very simple, but may represent some complicated dynamical properties. Most of the SI type models consist of the mass action principle, i.e., the assumption that the new cases arise in a simple proportion to the product of the number of individuals which are susceptible and the number of which are infectious. However, this principle has a limited validity and in the discrete models, this principle leads to biologically irrelevant results, unless some restrictions are suggested for the parameters. It is more appropriate for discrete epidemic models to include an exponential factor in the rate of transmission. Exponential difference equations can be used to study the models in population dynamics [1-3]. We consider here a simple exponential discrete-time host-pathogen model for spread of an infectious disease with permanent immunity. The time-step is equal to the duration of the infectious phase. The state variables are $S_{n}$, the number of susceptible individuals at time $n$, and $I_{n}$ representing the number of individuals, getting the disease (new cases) between times $n-1$

(c) 2013 Din et al.; licensee Springer. This is an Open Access article distributed under the terms of the Creative Commons Attribution License (http://creativecommons.org/licenses/by/2.0), which permits unrestricted use, distribution, and reproduction in any medium, provided the original work is properly cited. 
and $n$,

$$
I_{n+1}=S_{n}\left(1-e^{-\alpha I_{n}}\right), \quad S_{n+1}=S_{n} e^{-\alpha I_{n}}+\beta,
$$

where $\beta$ is the number of births between $n$ and $n+1$, all added to the susceptible class and assumed to be constant over time. So, the difference equation $S_{n+1}=S_{n} e^{-\alpha I_{n}}+\beta$ is just a 'conservation of mass' for the susceptible class. The first part $I_{n+1}=S_{n}\left(1-e^{-\alpha I_{n}}\right)$ of the model is just like Nicholson-Bailey; it comes from assuming that each susceptible escapes infection with probability $e^{-\alpha I_{n}}$; the more infectives there are, the lower the chance of escape. The model ignores mortality in the susceptible class, on the assumption that everyone gets the disease while young, and mortality occurs later in life.

Many ecological competition models are governed by differential and difference equations. We refer to $[4,5]$ and the references therein for some interesting results, related to the global character and local asymptotic stability. As it is pointed out in $[6,7]$, the discrete time models governed by difference equations are more appropriate than the continuous ones when the populations are of non-overlapping generations. The study of the discretetime models described by difference equations has now been given a great attention, since these models are more reasonable than the continuous time models when populations have non-overlapping generations. Discrete-time models give rise to more efficient computational models for numerical simulations and also show rich dynamics compared to the continuous ones. In recent years, many papers have been published on the mathematical models of biology that discussed the system of difference equations generated from the associated system of differential equations as well as the associated numerical methods. Mathematical models of epidemics have created a major area of research interest during the last few decades. Recently, theory on the effects of parasites on host population dynamics has received much attention and epidemiological models are often used to explain empirical results for host-parasites interaction system. For more details of such biological models, one can see [8-10].

More precisely, our aim is to investigate local asymptotic stability of unique positive equilibrium point, the global asymptotic character of equilibrium point, and the rate of convergence of positive solutions of system (1). For more results for the systems of difference equations, we refer the reader to [11-14].

\section{Boundedness and persistence}

The following theorem shows that every positive solution $\left\{\left(I_{n}, S_{n}\right)\right\}_{n=0}^{\infty}$ of system (1) is bounded and persists.

Theorem 1 Every positive solution $\left\{\left(I_{n}, S_{n}\right)\right\}$ of system (1) is bounded and persists.

Proof Let $\left\{\left(I_{n}, S_{n}\right)\right\}_{n=0}^{\infty}$ be any positive solution of system (1). It is easy to see that $S_{n} \geq \beta$ and $I_{n} \leq \beta$ for all $n=1,2, \ldots$. Then, it follows that $I_{n+1} \geq \beta\left(1-e^{-\alpha \beta}\right)$ and $S_{n+1} \leq S_{n} e^{-\alpha \beta\left(1-e^{-\alpha \beta}\right)}+\beta$. Consider the following difference equation

$$
z_{n+1}=z_{n} e^{-\alpha \beta\left(1-e^{-\alpha \beta}\right)}+\beta
$$


with initial condition $z_{0} \geq S_{0}$, then its solution is given by

$$
z_{n}=z_{0} A^{n-1}+\frac{\beta\left(1-A^{n}\right)}{1-A}
$$

where $A=e^{-\alpha \beta\left(1-e^{-\alpha \beta}\right)}$. Since $e^{-\alpha \beta\left(1-e^{-\alpha \beta}\right)}<1$, therefore,

$$
z_{n} \rightarrow \frac{\beta}{1-e^{-\alpha \beta\left(1-e^{-\alpha \beta}\right)}} \quad \text { as } n \rightarrow \infty
$$

Then, by comparison we have $S_{n} \leq z_{n} \leq \frac{\beta}{1-e^{-\alpha \beta\left(1-e^{-\alpha \beta}\right)}}$. Hence,

$$
\beta\left(1-e^{-\alpha \beta}\right) \leq I_{n} \leq \beta
$$

and

$$
\beta \leq S_{n} \leq \frac{\beta}{1-e^{-\alpha \beta\left(1-e^{-\alpha \beta}\right)}}
$$

for all $n=1,2, \ldots$.

Theorem 2 Let $\left\{\left(I_{n}, S_{n}\right)\right\}$ be a positive solution of the system (1). Then, $\left[\beta\left(1-e^{-\alpha \beta}\right), \beta\right] \times$ $\left[\beta, \frac{\beta}{1-e^{-\alpha \beta\left(1-e^{-\alpha \beta}\right)}}\right]$ is an invariant set for system (1).

Proof Let $\left\{\left(I_{n}, S_{n}\right)\right\}$ be a positive solution of system (1) with initial conditions $I_{0} \in I=[\beta(1-$ $\left.\left.e^{-\alpha \beta}\right), \beta\right]$ and $S_{0} \in J=\left[\beta, \frac{\beta}{1-e^{-\alpha \beta\left(1-e^{-\alpha \beta}\right)}}\right]$. Then, from system (1)

$$
I_{1}=S_{0}\left(1-e^{-\alpha I_{0}}\right) \geq \beta\left(1-e^{-\alpha \beta}\right)
$$

and

$$
I_{1}=S_{0}\left(1-e^{-\alpha I_{0}}\right) \leq \frac{\beta}{1-e^{-\alpha \beta\left(1-e^{-\alpha \beta}\right)}}\left(1-e^{-\alpha \beta\left(1-e^{-\alpha \beta}\right.}\right)=\beta .
$$

Similarly, we have

$$
S_{1}=S_{0} e^{-\alpha I_{0}}+\beta \geq \beta e^{-\alpha \beta}+\beta=\beta\left(1+e^{-\alpha \beta}\right) \geq \beta
$$

and

$$
S_{1}=S_{0} e^{-\alpha I_{0}}+\beta \leq \frac{\beta}{1-e^{-\alpha \beta\left(1-e^{-\alpha \beta}\right)}} e^{-\alpha \beta\left(1-e^{-\alpha \beta}\right)}+\beta \leq \frac{\beta}{1-e^{-\alpha \beta\left(1-e^{-\alpha \beta}\right)}} .
$$

Hence, $I_{1} \in I$ and $S_{1} \in J$. Suppose that the result is true for $n=k>1$, i.e., $I_{k} \in I$ and $S_{k} \in J$. Then from system (1), one can easily obtain

$$
\beta\left(1-e^{-\alpha \beta}\right) \leq I_{k+1} \leq \beta
$$

and

$$
\beta \leq S_{k+1} \leq \frac{\beta}{1-e^{-\alpha \beta\left(1-e^{-\alpha \beta}\right)}} .
$$

Hence, the proof is completed. 


\section{Linearized stability}

Let us consider two-dimensional discrete dynamical system of the form

$$
\begin{aligned}
& x_{n+1}=f\left(x_{n}, y_{n}\right), \\
& y_{n+1}=g\left(x_{n}, y_{n}\right), \quad n=0,1, \ldots,
\end{aligned}
$$

where $f: I \times J \rightarrow I$ and $g: I \times J \rightarrow J$ are continuously differentiable functions and $I, J$ are some intervals of real numbers. Furthermore, a solution $\left\{\left(x_{n}, y_{n}\right)\right\}_{n=0}^{\infty}$ of system (2) is uniquely determined by initial conditions $\left(x_{0}, y_{0}\right) \in I \times J$. An equilibrium point of (2) is a point $(\bar{x}, \bar{y})$ that satisfies

$$
\begin{aligned}
& \bar{x}=f(\bar{x}, \bar{y}), \\
& \bar{y}=g(\bar{x}, \bar{y}) .
\end{aligned}
$$

Let $(\bar{x}, \bar{y})$ be an equilibrium point of a map $F(x, y)=(f(x, y), g(x, y))$, where $f$ and $g$ are continuously differentiable functions at $(\bar{x}, \bar{y})$. The linearized system of (2) about the equilibrium point $(\bar{x}, \bar{y})$ is

$$
X_{n+1}=F\left(X_{n}\right)=F_{J} X_{n},
$$

where $X_{n}=\left(\begin{array}{l}x_{n} \\ y_{n}\end{array}\right)$ and $F_{J}$ is Jacobian matrix of system (2) about the equilibrium point $(\bar{x}, \bar{y})$. Let $(\bar{I}, \bar{S})$ be the equilibrium point of system (1), then one has

$$
\bar{I}=\bar{S}\left(1-e^{-\alpha \bar{I}}\right), \quad \bar{S}=\bar{S} e^{-\alpha \bar{I}}+\beta
$$

Then, it follows that $(\bar{I}, \bar{S})=\left(\beta, \frac{\beta}{1-e^{-\alpha \beta}}\right)$ is the unique positive equilibrium point of system (1). Moreover, the Jacobian matrix $F_{J}(\bar{I}, \bar{S})$ of system (1) about the equilibrium point $(\bar{I}, \bar{S})$ is given by

$$
F_{J}(\bar{I}, \bar{S})=\left(\begin{array}{cc}
\alpha \bar{S} e^{-\alpha \bar{I}} & 1-e^{-\alpha \bar{I}} \\
-\alpha \bar{S} e^{-\alpha \bar{I}} & e^{-\alpha \bar{I}}
\end{array}\right)
$$

The characteristic polynomial of $F_{J}(\bar{I}, \bar{S})$ is given by

$$
P(\lambda)=\lambda^{2}-e^{-\alpha \bar{I}}(1+\alpha \bar{S}) \lambda+\alpha \bar{S} e^{-\alpha \bar{I}} .
$$

Lemma 1 [15] Consider the second-degree polynomial equation

$$
\lambda^{2}+p \lambda+q=0
$$

where $p$ and $q$ are real numbers. Then, the necessary and sufficient condition for both roots of Equation (4) to lie inside the open disk $|\lambda|<1$ is

$$
|p|<1+q<2 \text {. }
$$


Lemma 2 [16] Assume that $X_{n+1}=F\left(X_{n}\right), n=0,1,2, \ldots$, is a system of difference equations and $\bar{X}$ is the fixed point of $F$. If all eigenvalues of the Jacobian matrix $J_{F}$ about $\bar{X}$ lie inside the open unit disk $|\lambda|<1$, then $\bar{X}$ is locally asymptotically stable. If one of them has a modulus greater than one, then $\bar{X}$ is unstable.

Theorem 3 Assume that $e^{\alpha \beta}(1+\alpha \beta)<\frac{1+e^{2 \alpha \beta}}{2}$. Then, the unique positive equilibrium point $(\bar{I}, \bar{S})=\left(\beta, \frac{\beta}{1-e^{-\alpha \beta}}\right)$ is locally asymptotically stable.

Proof The characteristic polynomial of $F_{J}(\bar{I}, \bar{S})$ about positive equilibrium point $\left(\beta, \frac{\beta}{1-e^{\alpha \beta}}\right)$ is given by

$$
P(\lambda)=\lambda^{2}-\left(e^{-\alpha \beta}+\frac{e^{-\alpha \beta} \alpha \beta}{1-e^{-\alpha \beta}}\right) \lambda+\frac{e^{-\alpha \beta} \alpha \beta}{1-e^{-\alpha \beta}} .
$$

Let

$$
f(\lambda)=\lambda^{2}, \quad g(\lambda)=\left(e^{-\alpha \beta}+\frac{e^{-\alpha \beta} \alpha \beta}{1-e^{-\alpha \beta}}\right) \lambda-\frac{e^{-\alpha \beta} \alpha \beta}{1-e^{-\alpha \beta}} .
$$

Assume that $e^{\alpha \beta}(1+\alpha \beta)<\frac{1+e^{2 \alpha \beta}}{2}$, and $|\lambda|=1$. Then, one has

$$
\begin{aligned}
|g(\lambda)| & \leq\left(e^{-\alpha \beta}+\frac{e^{-\alpha \beta} \alpha \beta}{1-e^{-\alpha \beta}}\right)+\frac{e^{-\alpha \beta} \alpha \beta}{1-e^{-\alpha \beta}} \\
& =e^{-\alpha \beta}+\frac{2 e^{-\alpha \beta} \alpha \beta}{1-e^{-\alpha \beta}} \\
& =\frac{e^{\alpha \beta}+2 \alpha \beta e^{\alpha \beta}-1}{e^{2 \alpha \beta}-e^{\alpha \beta}}<1 .
\end{aligned}
$$

Then, by Rouche's theorem $f(\lambda)$ and $f(\lambda)-g(\lambda)$ have the same number of zeroes in an open unit disk $|\lambda|<1$. Hence, both roots

$$
\lambda_{1}=\frac{1-e^{\alpha \beta}-e^{\alpha \beta} \alpha \beta+\sqrt{-4 e^{\alpha \beta}\left(-e^{\alpha \beta}+e^{2 \alpha \beta}\right) \alpha \beta+\left(1-e^{\alpha \beta}-e^{\alpha \beta} \alpha \beta\right)^{2}}}{2\left(e^{\alpha \beta}-e^{2 \alpha \beta}\right)}
$$

and

$$
\lambda_{2}=\frac{-1+e^{\alpha \beta}+e^{\alpha \beta} \alpha \beta+\sqrt{-4 e^{\alpha \beta}\left(-e^{\alpha \beta}+e^{2 \alpha \beta}\right) \alpha \beta+\left(1-e^{\alpha \beta}-e^{\alpha \beta} \alpha \beta\right)^{2}}}{2\left(-e^{\alpha \beta}+e^{2 \alpha \beta}\right)}
$$

of (5) lie in an open disk $|\lambda|<1$, and it follows from Lemma 2 that the equilibrium point $\left(\beta, \frac{\beta}{1-e^{-\alpha \beta}}\right)$ is locally asymptotically stable.

The following theorem shows the necessary and sufficient condition for the local asymptotic stability of a unique positive equilibrium point of system (1).

Theorem 4 The unique positive equilibrium point $(\bar{I}, \bar{S})=\left(\beta, \frac{\beta}{1-e^{-\alpha \beta}}\right)$ of system (1) is locally asymptotically stable if and only if $\frac{1+\alpha \beta}{e^{\alpha \beta}}<1$. 
Proof Let $p=-\left(e^{-\alpha \beta}+\frac{e^{-\alpha \beta} \alpha \beta}{1-e^{-\alpha \beta}}\right)$ and $q=\frac{e^{-\alpha \beta} \alpha \beta}{1-e^{-\alpha \beta}}$, then (5) can be written as

$$
P(\lambda)=\lambda^{2}+p \lambda+q
$$

Then, $|p|=e^{-\alpha \beta}+\frac{e^{-\alpha \beta} \alpha \beta}{1-e^{-\alpha \beta}}<1+\frac{e^{-\alpha \beta} \alpha \beta}{1-e^{-\alpha \beta}}=1+q$ and $1+q=1+\frac{e^{-\alpha \beta} \alpha \beta}{1-e^{-\alpha \beta}}<2$ if and only if $\frac{1+\alpha \beta}{e^{\alpha \beta}}<1$. Hence, from Lemma 1 , the unique positive equilibrium point $(\bar{I}, \bar{S})=\left(\beta, \frac{\beta}{1-e^{-\alpha \beta}}\right)$ of system (1) is locally asymptotically stable if and only if $\frac{1+\alpha \beta}{e^{\alpha \beta}}<1$.

\section{Global character}

The following lemma is similar to Theorem 1.16 of [15].

Lemma 3 Let $I=[a, b]$ and $J=[c, d]$ be real intervals, and let $f: I \times J \rightarrow I$ and $g: I \times J \rightarrow J$ be continuous functions. Consider system (2) with initial conditions $\left(x_{0}, y_{0}\right) \in I \times J$. Suppose that the following statements are true:

(i) $f(x, y)$ is non-decreasing in both arguments.

(ii) $g(x, y)$ is non-increasing in $x$, and non-decreasing in $y$.

(iii) If $\left(m_{1}, M_{1}, m_{2}, M_{2}\right) \in I^{2} \times J^{2}$ is a solution of the system

$$
\begin{array}{ll}
m_{1}=f\left(m_{1}, m_{2}\right), & M_{1}=f\left(M_{1}, M_{2}\right), \\
m_{2}=g\left(M_{1}, m_{2}\right), & M_{2}=g\left(m_{1}, M_{2}\right)
\end{array}
$$

such that $m_{1}=M_{1}$, and $m_{2}=M_{2}$.

Then, there exists exactly one equilibrium point $(\bar{x}, \bar{y})$ of the system (2) such that $\lim _{n \rightarrow \infty}\left(x_{n}, y_{n}\right)=(\bar{x}, \bar{y})$.

Theorem 5 The unique positive equilibrium point $(\bar{I}, \bar{S})=\left(\beta, \frac{\beta}{1-e^{-\alpha \beta}}\right)$ of system (1) is a global attractor.

Proof Let $f(x, y)=y\left(1-e^{-\alpha x}\right)$, and $g(x, y)=y e^{-\alpha x}+\beta$. Then, it is easy to see that $f(x, y)$ is non-decreasing in both $x$ and $y$. Moreover, $g(x, y)$ is non-increasing in $x$, and nondecreasing in $y$. Let $\left(m_{1}, M_{1}, m_{2}, M_{2}\right)$ be a solution of the system

$$
\begin{array}{ll}
m_{1}=f\left(m_{1}, m_{2}\right), & M_{1}=f\left(M_{1}, M_{2}\right), \\
m_{2}=g\left(M_{1}, m_{2}\right), & M_{2}=g\left(m_{1}, M_{2}\right) .
\end{array}
$$

Then, one has

$$
m_{1}=m_{2}\left(1-e^{-\alpha m_{1}}\right), \quad M_{1}=M_{2}\left(1-e^{-\alpha M_{1}}\right)
$$

and

$$
m_{2}=m_{2} e^{-\alpha M_{1}}+\beta, \quad M_{2}=M_{2} e^{-\alpha m_{1}}+\beta .
$$

From system (6), one has

$$
e^{-\alpha m_{1}}=\frac{m_{2}-m_{1}}{m_{2}}, \quad e^{-\alpha M_{1}}=\frac{M_{2}-M_{1}}{M_{2}} .
$$


From (7), one has

$$
e^{-\alpha m_{1}}=\frac{M_{2}-\beta}{M_{2}}, \quad e^{-\alpha M_{1}}=\frac{m_{2}-\beta}{m_{2}} .
$$

Furthermore, assuming as in the proof of Theorem 1.16 of [15], it suffices to suppose that

$$
m_{1} \leq M_{1}, \quad m_{2} \leq M_{2}
$$

Using the fact that $e^{-\alpha m_{1}} \geq e^{-\alpha M_{1}}$, one has from (8) and (9)

$$
\frac{m_{2}-m_{1}}{m_{2}} \geq \frac{m_{2}-\beta}{m_{2}}, \quad \frac{M_{2}-\beta}{M_{2}} \geq \frac{M_{2}-M_{1}}{M_{2}} .
$$

It follows from (10) that $m_{1} \leq \beta \leq M_{1}$. Then, (7) implies that

$$
M_{2}-m_{2}=\frac{\beta\left(e^{-\alpha m_{1}}-e^{-\alpha M_{1}}\right)}{\left(1-e^{-\alpha m_{1}}\right)\left(1-e^{-\alpha M_{1}}\right)}
$$

Using (6) in (11), we obtain

$$
\left|M_{2}-m_{2}\right|=\beta\left|\frac{M_{2}}{M_{1}}-\frac{m_{2}}{m_{1}}\right| .
$$

Following the same technique as in the proof of Proposition 4.1 of [17], one has $\left|M_{2}-m_{2}\right| \leq$ $\frac{\beta}{M_{1}}\left|M_{2}-m_{2}\right|$, i.e., $\left(1-\frac{\beta}{M_{1}}\right)\left|M_{2}-m_{2}\right| \leq 0$. Thus, $m_{2}=M_{2}$ and, similarly, one can show that $m_{1}=M_{1}$. Hence, from Lemma 3 , the equilibrium point $\left(\beta, \frac{\beta}{1-e^{-\alpha \beta}}\right)$ of system $(1)$ is a global attractor.

Lemma 4 The unique positive equilibrium point $(\bar{I}, \bar{S})=\left(\beta, \frac{\beta}{1-e^{\alpha \beta}}\right)$ of system (1) is globally asymptotically stable if and only if $\frac{1+\alpha \beta}{e^{\alpha \beta}}<1$.

Proof The proof follows from Theorem 4 and Theorem 5.

\section{Rate of convergence}

In this section, we determine the rate of convergence of a solution that converges to the unique positive equilibrium point of system (1). Similar methods can be found in [18] and [19].

The following result gives the rate of convergence of solutions of a system of difference equations

$$
X_{n+1}=(A+B(n)) X_{n},
$$

where $X_{n}$ is an $m$-dimensional vector, $A \in C^{m \times m}$ is a constant matrix, and $B: \mathbb{Z}^{+} \rightarrow C^{m \times m}$ is a matrix function satisfying

$$
\|B(n)\| \rightarrow 0
$$

as $n \rightarrow \infty$, where $\|\cdot\|$ denotes any matrix norm, which is associated with the vector norm

$$
\|(x, y)\|=\sqrt{x^{2}+y^{2}}
$$


Proposition 1 (Perron's theorem [20]) Suppose that condition (13) holds. If $X_{n}$ is a solution of (12), then either $X_{n}=0$ for all large $n$ or

$$
\rho=\lim _{n \rightarrow \infty}\left(\left\|X_{n}\right\|\right)^{1 / n}
$$

exists and is equal to the modulus of one the eigenvalues of matrix $A$.

Proposition 2 [20] Suppose that condition (13) holds. If $X_{n}$ is a solution of (12), then either $X_{n}=0$ for all large $n$ or

$$
\rho=\lim _{n \rightarrow \infty} \frac{\left\|X_{n+1}\right\|}{\left\|X_{n}\right\|}
$$

exists and is equal to the modulus of one the eigenvalues of matrix $A$.

Let $\left\{\left(I_{n}, S_{n}\right)\right\}$ be any solution of system (1) such that $\lim _{n \rightarrow \infty} I_{n}=\bar{I}$, and $\lim _{n \rightarrow \infty} S_{n}=\bar{S}$. To find the error terms, one has from system (1)

$$
I_{n+1}-\bar{I}=S_{n}\left(1-e^{-\alpha I_{n}}\right)-\bar{S}\left(1-e^{-\alpha \bar{I}}\right)
$$

and

$$
S_{n+1}-\bar{S}=S_{n} e^{-\alpha I_{n}}-\bar{S} e^{-\alpha \bar{I}}
$$

Let $e_{n}^{1}=I_{n}-\bar{I}$, and let $e_{n}^{2}=S_{n}-\bar{S}$, then one has

$$
e_{n+1}^{1}=a_{n} e_{n}^{1}+b_{n} e_{n}^{2}
$$

and

$$
e_{n+1}^{2}=c_{n} e_{n}^{1}+d_{n} e_{n}^{2}
$$

where

$$
\begin{array}{ll}
a_{n}=\frac{\bar{S}\left(e^{-\alpha \bar{I}}-e^{-\alpha I_{n}}\right)}{I_{n}-\bar{I}}, & b_{n}=1-e^{-\alpha I_{n},} \\
c_{n}=\frac{\bar{S}\left(e^{-\alpha I_{n}}-e^{-\alpha \bar{I}}\right)}{I_{n}-\bar{I}}, & d_{n}=e^{-\alpha I_{n}} .
\end{array}
$$

Moreover,

$$
\begin{array}{ll}
\lim _{n \rightarrow \infty} a_{n}=\alpha \bar{S} e^{-\alpha \bar{I}}, & \lim _{n \rightarrow \infty} b_{n}=1-e^{-\alpha \bar{I}}, \\
\lim _{n \rightarrow \infty} c_{n}=-\alpha \bar{S} e^{-\alpha \bar{I}}, & \lim _{n \rightarrow \infty} d_{n}=e^{-\alpha \bar{I}} .
\end{array}
$$

Now, the limiting system of error terms can be written as

$$
\left[\begin{array}{c}
e_{n+1}^{1} \\
e_{n+1}^{2}
\end{array}\right]=\left[\begin{array}{cc}
\alpha \bar{S} e^{-\alpha \bar{I}} & 1-e^{-\alpha \bar{I}} \\
-\alpha \bar{S} e^{-\alpha \bar{I}} & e^{-\alpha \bar{I}}
\end{array}\right]\left[\begin{array}{l}
e_{n}^{1} \\
e_{n}^{2}
\end{array}\right],
$$

which is similar to linearized system of (1) about the equilibrium point $(\bar{I}, \bar{S})$. 
Using Proposition 1, one has the following result.

Theorem 6 Assume that $\left\{\left(I_{n}, S_{n}\right)\right\}$ is a positive solution of system (1) such that $\lim _{n \rightarrow \infty} I_{n}=$ $\bar{I}$ and $\lim _{n \rightarrow \infty} S_{n}=\bar{S}$, where $(\bar{I}, \bar{S})$ is a unique positive equilibrium point of (1). Then, the error vector $e_{n}=\left(\begin{array}{c}e_{n}^{1} \\ e_{n}^{2}\end{array}\right)$ of every solution of (1) satisfies both of the following asymptotic relations

$$
\lim _{n \rightarrow \infty}\left(\left\|e_{n}\right\|\right)^{\frac{1}{n}}=\left|\lambda_{1,2} F_{J}(\bar{I}, \bar{S})\right|, \quad \lim _{n \rightarrow \infty} \frac{\left\|e_{n+1}\right\|}{\left\|e_{n}\right\|}=\left|\lambda_{1,2} F_{J}(\bar{I}, \bar{S})\right|,
$$

where $\lambda_{1,2} F_{J}(\bar{I}, \bar{S})$ are the characteristic roots of the Jacobian matrix $F_{J}(\bar{I}, \bar{S})$.

\section{Examples}

In order to verify our theoretical results and to support our theoretical discussions, we consider several interesting numerical examples in this section. These examples represent different types of qualitative behavior of solutions to the system of nonlinear difference equations (1). All plots in this section are drawn with Mathematica.

Example 1 Let $\alpha=0.05$, and let $\beta=0.3$. Then, system (1) can be written as

$$
x_{n+1}=y_{n}\left(1-e^{-0.05 x_{n}}\right), \quad y_{n+1}=y_{n} e^{-0.05 x_{n}}+0.3,
$$

with initial conditions $x_{0}=0.2, y_{0}=20$.

In this case, the unique equilibrium point $\left(\beta, \frac{\beta}{1-e^{-\alpha \beta}}\right)=(0.3,20.1504)$. Moreover, in Figure 1, the plot of $x_{n}$ is shown in Figure 1(a), the plot of $y_{n}$ is shown in Figure 1(b), and an attractor of system (16) is shown in Figure 1(c). The basic reproductive number of system (16) is $R_{0}=\frac{1+\alpha \beta}{e^{\alpha \beta}}=0.999889<1$.

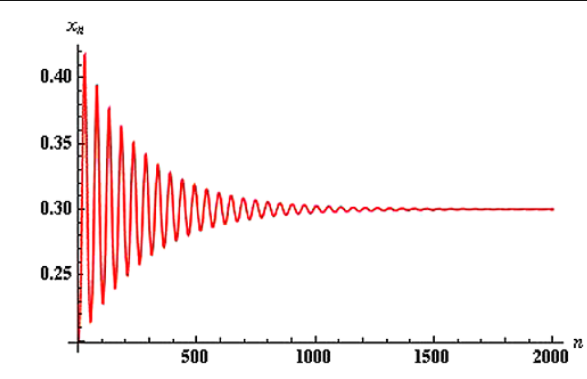

(a) Plot of $x_{n}$ for system (16).

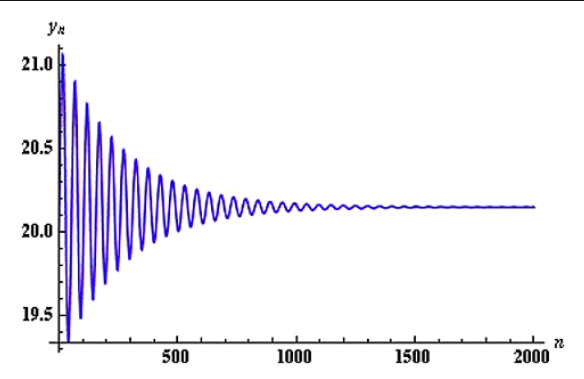

(b) Plot of $y_{n}$ for system (16).

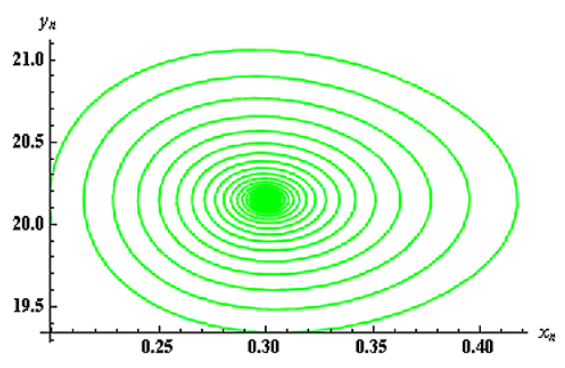

(c) An attractor of system (16).

Figure 1 Plots for system (16). 


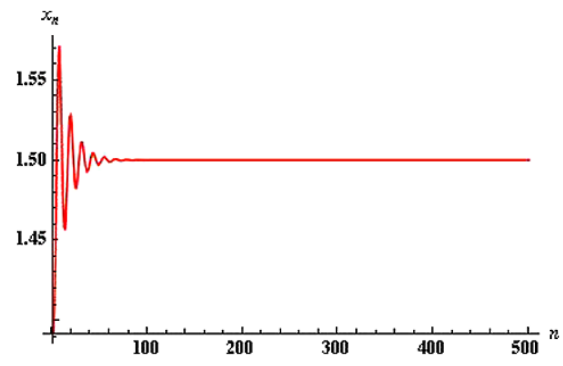

(a) Plot of $x_{n}$ for system (17).

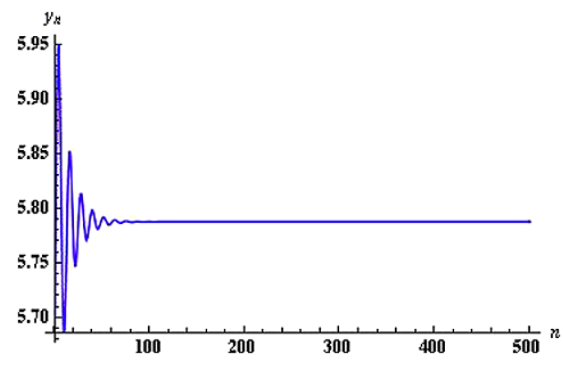

(b) Plot of $y_{n}$ for system (17).

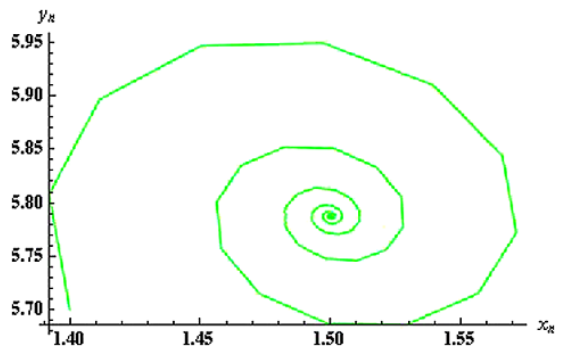

(c) An attractor of system (17).

Figure 2 Plots for system (17).

Example 2 Let $\alpha=0.2$, and let $\beta=1.5$. Then, system (1) can be written as

$$
x_{n+1}=y_{n}\left(1-e^{-0.2 x_{n}}\right), \quad y_{n+1}=y_{n} e^{-0.2 x_{n}}+1.5,
$$

with initial conditions $x_{0}=1.4, y_{0}=5.7$.

In this case, the unique equilibrium point $\left(\beta, \frac{\beta}{1-e^{-\alpha \beta}}\right)=(1.5,5.78744)$. Moreover, in Figure 2, the plot of $x_{n}$ is shown in Figure 2(a), the plot of $y_{n}$ is shown in Figure 2(b), and an attractor of system (16) is shown in Figure 2(c). The basic reproductive number of system (17) is $R_{0}=\frac{1+\alpha \beta}{e^{\alpha \beta}}=0.963064<1$.

Example 3 Let $\alpha=0.9$, and let $\beta=0.01$. Then, system (1) can be written as

$$
x_{n+1}=y_{n}\left(1-e^{-0.9 x_{n}}\right), \quad y_{n+1}=y_{n} e^{-0.9 x_{n}}+0.01,
$$

with initial conditions $x_{0}=0.1, y_{0}=1.1$.

In this case, the unique equilibrium point $\left(\beta, \frac{\beta}{1-e^{-\alpha \beta}}\right)=(0.01,1.11612)$. Moreover, in Figure 3, the plot of $x_{n}$ is shown in Figure 3(a), the plot of $y_{n}$ is shown in Figure 3(b), and an attractor of system (18) is shown in Figure 3(c). The basic reproductive number of system (18) is $R_{0}=\frac{1+\alpha \beta}{e^{\alpha \beta}}=0.99996<1$.

Example 4 Let $\alpha=0.01$, and let $\beta=0.05$. Then, system (1) can be written as

$$
x_{n+1}=y_{n}\left(1-e^{-0.01 x_{n}}\right), \quad y_{n+1}=y_{n} e^{-0.01 x_{n}}+0.05 \text {, }
$$

with the initial conditions $x_{0}=0.02, y_{0}=100$.

In this case, the unique equilibrium point $\left(\beta, \frac{\beta}{1-e^{-\alpha \beta}}\right)=(0.05,100.025)$. Moreover, in Figure 4, the plot of $x_{n}$ is shown in Figure 4(a), the plot of $y_{n}$ is shown in Figure 4(b), and an 


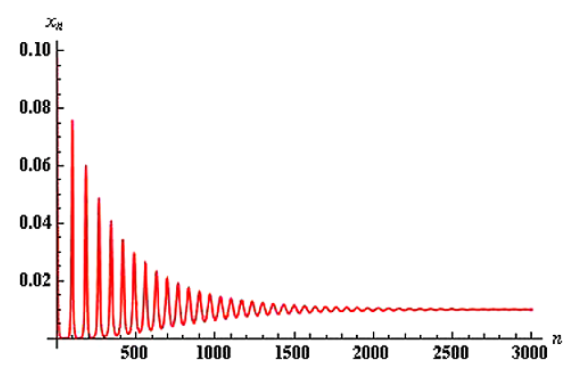

(a) Plot of $x_{n}$ for system (18)

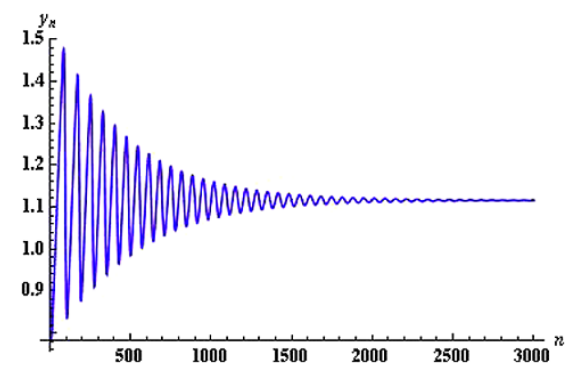

(b) Plot of $y_{n}$ for system (18).

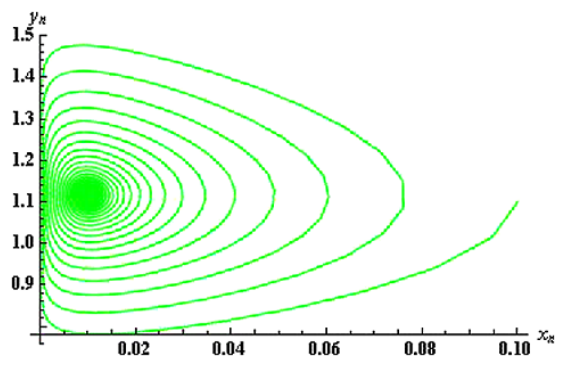

(c) An attractor of system (18).

Figure 3 Plots for system (18).

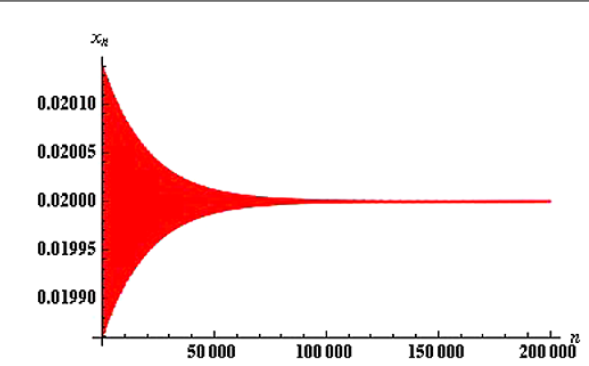

(a) Plot of $x_{n}$ for system (19).

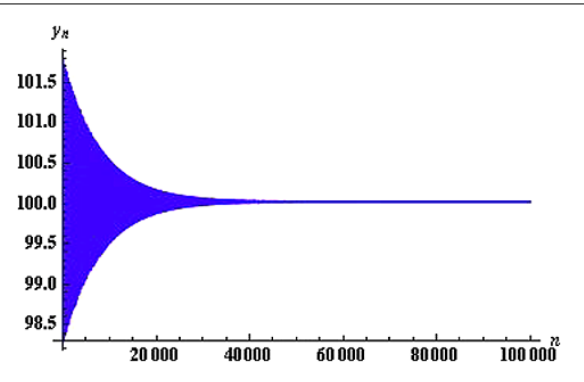

(b) Plot of $y_{n}$ for system (19).

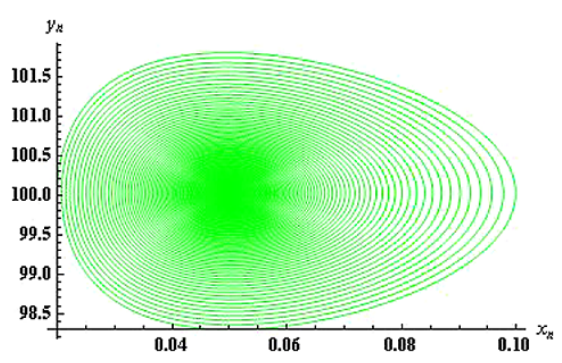

(c) An attractor of system (19).

Figure 4 Plots for system (19). 


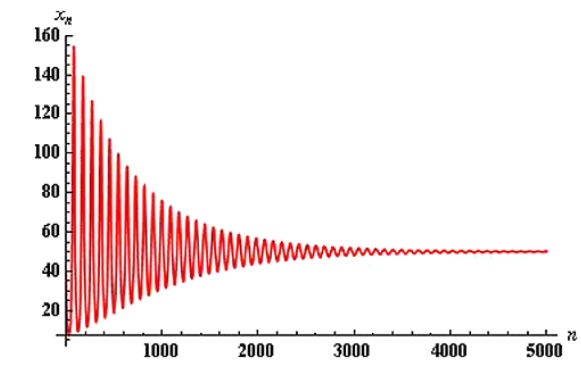

(a) Plot of $x_{n}$ for system (20).

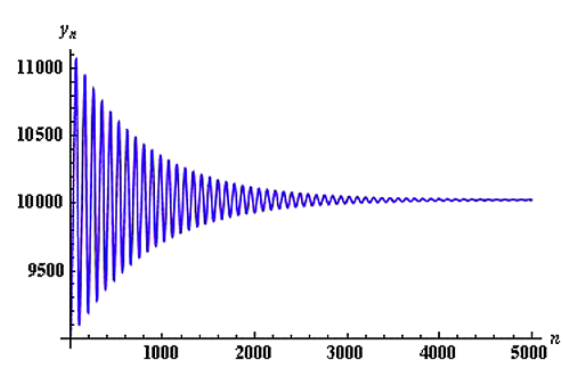

(b) Plot of $y_{n}$ for system (20).

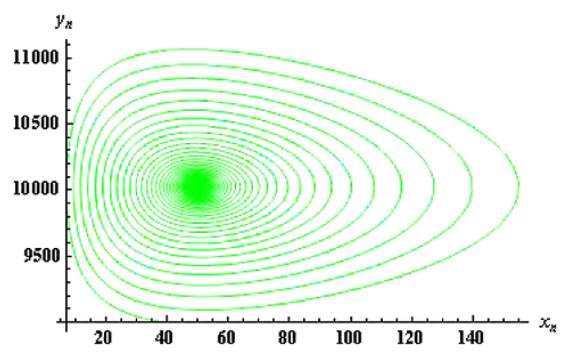

(c) An attractor of system (20).

Figure 5 Plots for system (20).

attractor of the system (19) is shown in Figure 4(c). The basic reproductive number of system (19) is $R_{0}=\frac{1+\alpha \beta}{e^{\alpha \beta}}=0.9999998750416589<1$.

Example 5 Let $\alpha=0.0001$, and let $\beta=50$. Then, system (1) can be written as

$$
x_{n+1}=y_{n}\left(1-e^{-0.0001 x_{n}}\right), \quad y_{n+1}=y_{n} e^{-0.0001 x_{n}}+50 \text {, }
$$

with the initial conditions $x_{0}=40, y_{0}=9,000$.

In this case, the unique equilibrium point $\left(\beta, \frac{\beta}{1-e^{-\alpha \beta}}\right)=(50,10,025)$. Moreover, in Figure 5, the plot of $x_{n}$ is shown in Figure 5(a), the plot of $y_{n}$ is shown in Figure 5(b), and an attractor of system (20) is shown in Figure 5(c). The basic reproductive number of system (20) is $R_{0}=\frac{1+\alpha \beta}{e^{\alpha \beta}}=0.9999875415886458<1$.

\section{Conclusion and future work}

This work is related to the qualitative behavior of an exponential discrete-time hostpathogen model for spread of an infectious disease with permanent immunity. We proved that system (1) has a unique positive equilibrium point, which is locally asymptotically stable. The main objective of dynamical systems theory is to predict the global behavior of a system based on the knowledge of its present state. An approach to this problem consists of determining the possible global behaviors of the system and determining which initial conditions lead to these long-term behaviors. In the paper, a general result for global character for such type of systems is proved. Due to the simplicity of our SI-type model, we have carried out a systematic local and global stability analysis of it. The most important finding here is that the unique positive equilibrium point can be a global asymptotic attractor for model (1). Moreover, the rate convergence of positive solutions has also been investigated. In such models, there is a threshold parameter that might tell whether a population will increase or die out, or whether an infectious disease will persist or die out 
within a population. This parameter is commonly known as the basic reproductive number and is denoted by $R_{0}$. In epidemiology, this number $R_{0}$ is defined as the number of newly infected individual, produced by a single infected individual in its period of infectivity. In case of system (1), the basic reproductive number is given by $R_{0}=\frac{1+\alpha \beta}{e^{\alpha \beta}}$. From our investigations, it is obvious that the unique positive equilibrium point of system (1) is globally asymptotically stable if $R_{0}<1$, and unstable if $R_{0}>1$. Some numerical examples are provided to support our theoretical results. These examples are experimental verifications of theoretical discussions. The qualitative behavior of the general model, where there is host mortality at some constant rate, will be our next aim to study.

\section{Competing interests}

The authors declare that they have no competing interests.

\section{Authors' contributions}

All authors contributed equally and approved the final version of the manuscript.

\section{Acknowledgements}

The authors would like to thank the anonymous referees for their valuable comments and suggestions leading to improvement of this paper. This work was supported by the Higher Education Commission of Pakistan.

Received: 23 April 2013 Accepted: 29 July 2013 Published: 29 August 2013

\section{References}

1. El-Metwally, E, Grove, EA, Ladas, G, Levins, R, Radin, M: On the difference equation $x_{n+1}=\alpha+\beta x_{n-1} e^{-x_{n}}$. Nonlinear Anal. 47, 4623-4634 (2001)

2. Papaschinopoulos, G, Radin, MA, Schinas, CJ: On the system of two difference equations of exponential form: $x_{n+1}=a+b x_{n-1} e^{-y_{n}}, y_{n+1}=c+d y_{n-1} e^{-x_{n}}$. Math. Comput. Model. 54, 2969-2977 (2011)

3. Papaschinopoulos, G, Schinas, CJ: On the dynamics of two exponential type systems of difference equations. Comput. Math. Appl. 64(7), 2326-2334 (2012)

4. Ahmad, S: On the nonautonomous Lotka-Volterra competition equation. Proc. Am. Math. Soc. 117, $199-204$ (1993)

5. Tang, X, Zou, X: On positive periodic solutions of Lotka-Volterra competition systems with deviating arguments. Proc. Am. Math. Soc. 134, 2967-2974 (2006)

6. Zhou, Z, Zou, X: Stable periodic solutions in a discrete periodic logistic equation. Appl. Math. Lett. 16(2), 165-171 (2003)

7. Liu, X: A note on the existence of periodic solution in discrete predator-prey models. Appl. Math. Model. 34, 2477-2483 (2010)

8. Allen, LJS: An Introduction to Mathematical Biology. Prentice Hall, New York (2007)

9. Brauer, F, Castillo-Chavez, C: Mathematical Models in Population Biology and Epidemiology. Springer, Berlin (2000)

10. Edelstein-Keshet, L: Mathematical Models in Biology. McGraw-Hill, New York (1988)

11. Kalabusisć, S, Kulenović, MRS, Pilav, E: Dynamics of a two-dimensional system of rational difference equations of Leslie-Gower type. Adv. Differ. Equ. (2011). doi:10.1186/1687-1847-2011-29

12. Din, Q: Dynamics of a discrete Lotka-Volterra model. Adv. Differ. Equ. 2013, 95 (2013)

13. Din, Q, Donchev, T: Global character of a host-parasite model. Chaos Solitons Fractals 54, 1-7 (2013)

14. Din, Q, Qureshi, MN, Khan, AQ: Dynamics of a fourth-order system of rational difference equations. Adv. Differ. Equ. 2012, 215 (2012)

15. Grove, EA, Ladas, G: Periodicities in Nonlinear Difference Equations. Chapman \& Hall/CRC Press, Boca Raton (2004)

16. Sedaghat, H: Nonlinear Difference Equations: Theory with Applications to Social Science Models. Kluwer Academic, Dordrecht (2003)

17. Papaschinopoulos, G, Radin, MA, Schinas, CJ: Study of the asymptotic behavior of the solutions of three systems of difference equations of exponential form. Appl. Math. Comput. 218, 5310-5318 (2012)

18. Kulenović, MRS, Nurkanović, M: Asymptotic behavior of a competitive system of linear fractional difference equations. Adv. Differ. Equ. 2006, Article ID 019756 (2006)

19. Clark, CA, Kulenović, MRS, Selgrade, JF: On a system of rational difference equations. J. Differ. Equ. Appl. 11(7), 565-580 (2005)

20. Pituk, M: More on Poincare's and Perron's theorems for difference equations. J. Differ. Equ. Appl. 8, 201-216 (2002)

doi:10.1186/1687-1847-2013-263

Cite this article as: Din et al.: Qualitative behavior of a host-pathogen model. Advances in Difference Equations 2013 2013:263. 